\title{
Restos de Anfibios en la Costa de la Araucanía: Problematización y Perspectivas
}

\author{
Amphibians Remains in the Araucanian Coast: Problematization and Prospects \\ Daniel Quiroz L. ${ }^{i}$ e Ismael Martínez R ${ }^{\mathrm{ii}}$.
}

\begin{abstract}
RESUMEN
Los restos de anfibios no han sido mayormente considerados en los trabajos arqueológicos publicados sobre la zona costera que se extiende entre el río Bío Bío y el canal de Chacao, existiendo solo mínimas menciones en los conjuntos faunísticos analizados. El hallazgo recurrente de restos de anfibios en una serie de sitios arqueológicos, tanto continentales como insulares de las costas de Arauco, nos conduce a investigar la naturaleza de las causas que explican su presencia o ausencia en los sitios arqueológicos estudiados, en relación con las poblaciones humanas en la Araucanía. Este trabajo se enfoca en la presentación de los diversos problemas zooarqueológicos, asociados tanto con la identificación anatómica y taxonómica de restos de anfibios, como con la naturaleza de los agentes tafonómicos que actúan sobre dichos restos.
\end{abstract}

Palabras Clave: Huesos de Anfibios, Consumo Humano, Complejo El Vergel, Centro Sur de Chile.

ABSTRACT

The remains of amphibians have not been mostly considered in the archaeological literature on the coastal zone between the Bío Bío River and the Chacao channel.The finding of remains of amphibians in continental and insular archaeological sites of Arauco coast, led us to research the causes that explain their presence or absence in the archaeological sites in relation to the human populations of Araucanía. This paper focuses on the different zooarchaeological issues, associated with anatomical and taxonomic identification of Amphibians remains, and the nature of taphonomic agents that act on these remains.

Keywords: Amphibians Remains, Human Consumption, El Vergel Complex, Central-South Chile.

${ }^{i}$ Centro de Documentación de Bienes Patrimoniales, Dirección de Bibliotecas, Archivos y Museos. Recoleta 683, Santiago, Chile. Correo-e: dquiroz@cdbp.cl.

ii Profesional Independiente. Correo-e: ismart68@gmail.com

Recibido: 04-01-2013 - Revisado: 20-12-2013 - Aceptado: 28-02-2014 


\section{INTRODUCCIÓN}

Este trabajo aborda parte de la fauna menor, taxones cuya masa no sobrepasa los $5 \mathrm{~kg}$. (Andrews 1990). En particular, se aboca a los anfibios, los que comúnmente gozan de poca atención en los estudios zooarqueológicos (O'Connor 2000, Kiselý 2008), pero que, en estudios recientes en Chile, han sobrepasado mayoritariamente a los taxones mayores (Bastías 20II).

Los restos de anfibios no han sido estudiados con la suficiente profundidad en los trabajos realizados en Chile. Revisando los trabajos arqueológicos publicados sobre la zona costera que se extiende entre el río Bío Bío y el canal de Chacao, tenemos que casi no se menciona la presencia de restos de anfibios como parte de los conjuntos faunísticos (Velásquez y Adan 2002). Un reciente estudio aborda este problemática en un sitio de Chile Central (Bastías 20II).

De igual modo, en la literatura especializada, los huesos de anfibios han sido reportados sin mayor caracterización, donde su presencia no se explica en términos culturales (Stahl 2000, Yerkes 2005), o bien se reconoce inexperiencia en este tipo de fauna (Klein 1979).

Los restos de anfibios pueden ser depositados tanto por procesos culturales (Kiselý 2008), como naturales (Lucas et al 2010); ya sea por acumulación de depredadores (Andrews 1995), o bien corresponder a ejemplares que se entrampan en pozos (Stahl 2000, Yerkes 2005), y/o mueren en forma natural (Sampson 2003). Es por esto que resulta esencial investigar los procesos que estarían incidiendo para comprender su ingreso al registro (Pinto y Andrews 1999).

\section{BIOLOGÍA}

La taxonomía y sistemática de la Clase Amphibia contempla la existencia de tres Ordenes, Anura (sapos y ranas), Caudata (salamandras y tritones) y Gymnophiona (cecilias o culebras ciegas). Mientras que la batracofauna chilena, contempla solamente la presencia de anuros, cuya taxa se encuentra razonablemente bien establecida (Cei 1962, Formas 1995). En Chile hay presentes 6 familias, Calyptocephalellidae, Bufonidae, Cerathophrydae,
Cycloramphidae, Leiuperidae y Pipidae (exótica), I5 géneros y 57 especies de anfibios (Frost 2004, Díaz y Ortiz 2003, Díaz 2005).

Los anfibios son los tetrápodos más antiguos y forman el tronco basal del que han derivado todos los demás vertebrados terrestres. Tienen esqueletos simples, con bastante menos huesos que otros vertebrados modernos y muchos menos que sus antepasados pisciformes. Esto denota una tendencia evolutiva o un cambio rotundo en los anfibios hacia una reducción del número de huesos en el cráneo y de vértebras en la columna vertebral (Roček 2003, Ročkova y Roček 2005). Los anuros se caracterizan por tener una cabeza ancha, amplias órbitas oculares y una columna vertebral corta, usualmente sin costillas; las vértebras caudales se encuentran fusionadas en un hueso denominado urostilo (Duellman y Trueb 1986); tienen huesos fusionados denominados radioulna (radio+ulna) y tibiofíbula (tibia+fíbula); tanto el tibial como el fibular son muy elongados, lo que permite un soporte adicional que los anuros utilizan para saltar (Gans and Parsons 1966).

Calyptocephalellidae es una familia de anfibios endémica de Chile y está representado por dos géneros Calyptocephalella, con una sola especie, C. gayi, y Telmatobufo, con tres especies, T. australis, $T$. bullocki y $T$. venustus.

\section{LOS SITIOS}

La presencia recurrente de restos de anfibios en una serie de sitios arqueológicos, tanto continentales como insulares, en las costas de Arauco nos ha llevado a investigar las causas que explican su presencia o ausencia en los sitios arqueológicos estudiados. Los ejemplares provienen de excavaciones sistemáticas en seis sitios arqueológicos, cuatro continentales, Lenga 2 (LG-2), El Arenal I (EA-I), Raqui-Tubul 39 (RT-39) y Quiapo I (QP-I), dos insulares, SM-6 (Isla Santa María) y P2II (Isla Mocha), la mayoría en el marco del Proyecto Fondecyt 1020272.

El ambiente en el que se encuentran estos sitios, excepto P2I-I, corresponde a humedales, es decir, lagunas, vegas, ríos y/o esteros, rodeados por cordones de dunas, cercanos al mar. La matriz de los sitios es arenosa. Las ocupaciones de estos sitios se 
inician hacia el 400 d.C. para finalizar el 1600 d.C., aunque, probablemente continuaran hasta épocas más tardías. Corresponden culturalmente a lo que se ha denominado Complejo Arqueológico El Vergel (Quiroz 2003).

Aunque el porcentaje de restos de anfibios presentes no es muy significativo respecto de conjunto faunístico total (excepto tal vez en EA-I y RT-39), resulta igualmente relevante cuestionarse por su presencia/ausencia en los contextos arqueológicos.

\section{MÉTODO}

Los restos de anfibios provenientes de excavaciones arqueológicas realizadas en la zona de Concepción y Arauco, recuperados con una malla de $5 \mathrm{~mm}$ y depositados en el Museo de Historia Natural de Concepción, son analizados considerando la información biológica, tafonómica y cultural contenida en ellos. Fueron estudiados, primero, individualmente, es decir, registrando la información proveniente de cada fragmento o espécimen presente (Reitz \&Wing
1999), comparados con las colecciones de referencia del Museo Nacional de Historia Natural (Santiago, Chile) y luego examinados con una lupa de 10x y $20 x$, originando una base de datos que permitiera tener un control más riguroso sobre el conjunto óseo. Luego, los restos fueron estudiados en su conjunto, para lograr sistematizar la información zooarqueológica, representar adecuadamente el conjunto óseo y hacerlo comparable con otros conjuntos provenientes de otros contextos. Se han empleado como indicadores el NISP [número de especímenes óseos identificados por taxón] y el MNE [número mínimo de elementos].

\section{RESULTADOS}

Los restos de anfibios recuperados corresponden a diversas unidades anatómicas (Figura I), cuya distribución en los sitios se muestra en la Tabla I. Todos los fragmentos óseos pudieron ser asignados a la especie Calyptocephalella gayi [Dumeril \& Bibron 184I] (Anura, Calyptocephalellidae, rana grande chilena o helmeted water toad], antiguamente Caudiverbera caudiverbera (Myers \& Stothers 2006).

\begin{tabular}{|l|l|l|l|l|l|l|l|l|l|l|l|l|l|l|}
\hline & LG-2 & $\begin{array}{c}400- \\
900 \\
\text { d.C. }\end{array}$ & EA-I & $\begin{array}{c}970- \\
\text { I425 } \\
\text { d.C. }\end{array}$ & $\begin{array}{c}\text { RT } \\
39\end{array}$ & $\begin{array}{c}650- \\
850 \\
\text { d.C. }\end{array}$ & QP-I & $\begin{array}{c}\text { Sin } \\
\text { fechas }\end{array}$ & SM-6 & $\begin{array}{c}970- \\
\text { I425 } \\
\text { d.C. }\end{array}$ & $\begin{array}{c}\text { P2I- } \\
\text { I }\end{array}$ & $\begin{array}{c}1000- \\
\text { I600 } \\
\text { d.C. }\end{array}$ & \\
\hline NISP & F & $\%$ & $F$ & $\%$ & $F$ & $\%$ & $F$ & $\%$ & $F$ & $\%$ & F & $\%$ & TOTAL & $\%$ \\
\hline Aves & 409 & 15,9 & 183 & 25 & 8 & 8,9 & 73 & 25,0 & 48 & 10,4 & 356 & 49,3 & 1077 & 22,1 \\
\hline Anfibios & 25 & 1 & 31 & 4,2 & 24 & 26,7 & 4 & 1,4 & 6 & 1,3 & 0 & 0 & 90 & 1,8 \\
\hline Mamíferos & 1.820 & 70,6 & 310 & 42,3 & 12 & 13,3 & 114 & 39,0 & 265 & 57,4 & 225 & 31,2 & 2746 & 56,3 \\
\hline Peces & 323 & 12,5 & 209 & 28,5 & 46 & 51,1 & 101 & 34,6 & 143 & 30,9 & 141 & 19,5 & 963 & 19,7 \\
\hline TOTAL & 2.577 & 100 & 733 & 100 & 90 & 100 & 292 & 100 & 462 & 100 & 722 & 100 & 4.876 & 100 \\
\hline
\end{tabular}

Tabla 1: restos de anfibios por cada sitio arqueológico. LG-2 = Lenga2, EA-1 = El Arenal 1, RT-39= Raqui-Tubul 39, QP-1 = Quiapo 1 , SM-6 = Isla Santa María y P21-1 = Isla Mocha

Table 1: amphibian remains of each archaeological site. $L G-2=$ Lenga2, EA-1 = El Arenal 1, RT-39= Raqui-Tubul 39, QP-1 = Quiapo 1 , SM-6 = Isla Santa María y P21-1 = Isla Mocha 

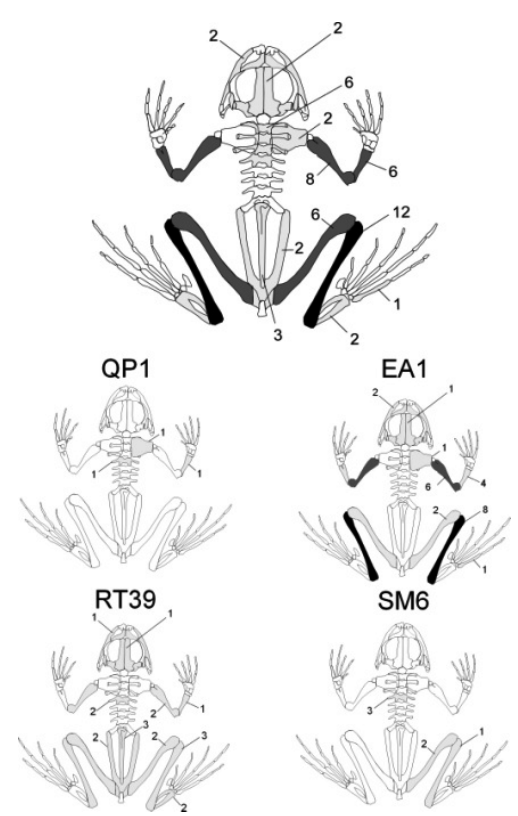

Figura 1: Representación de elementos anatómicos (NISP). EA-1 = El Arenal 1, RT-39 = Raqui-Tubul 39, QP-1 = Quiapo 1 y SM-6 = Isla Santa María

Figure 1: The representation of anatomical elements $E A-1=E l$ Arenal 1, RT-39= Raqui-Tubul 39, QP-1 = Quiapo 1 y SM-6 = Isla Santa María

C. gayi es un anfibio de gran tamaño, los machos pueden llegar a medir $120 \mathrm{~mm}$ y las hembras $320 \mathrm{~mm}$, con cuerpo robusto, cabeza ancha y muy grande en la base, hocico corto y redondo, ojos pequeños en relación a la cabeza. Piel lisa y verdosa manchada irregularmente, vientre blanco o amarillento. Su peso normal es de $0.5 \mathrm{~kg}$, pero algunos ejemplares pueden llegar a los $3 \mathrm{~kg}$ (Duellmann 2003). Se distribuye desde Coquimbo hasta Puerto Montt. Se encuentra principalmente en lagos, lagunas, arroyos y estanques que presenten orillas suaves con poca pendiente y abundante vegetación acuática. Su hábitat típico corresponde a aguas quietas e incluso a remansos en las aguas corrientes. Se alimentan de crustáceos, peces, pequeños roedores e incluso de otros anfibios (Cei 1962).
Los restos óseos de anfibios han sido abordados en términos tafonómicos por la experimentación actualística y los análisis de laboratorio (Andrews 1995, Pinto y Andrews 1999). Al igual que para los micromamíferos, la investigación de los procesos tafonómicos de los anfibios a sido desarrollada principalmente mediante el estudio de fecas o egagrópilas de aves y la variación de los ácidos gástricos de sus depredadores (Andrews 1990, Pinto y Andrews 1999).

En nuestro conjunto, proveniente de sitios a cielo abierto, no fueron detectados restos provenientes de fecas o egagrópilas de aves. De igual modo, a escala macroscópica y observación con lupa de mano de 20X, no se observaron huellas de ácidos digestivos (Andrews 1990).

Las principales alteraciones registradas en los huesos corresponden a bioerosión (sensu Davis 1997), la que consideramos que no siempre es atribuible a la acción de raíces. En este sentido, se detectaron Túneles Hackett (Hackett 198I, retomados por Davis 1997), los que se caracterizan por configurar una red de túneles en la superficie del hueso, configurando un patrón meándrico característico que, para ambientes marinos o lacustres, serían fruto de cynobacterias o algas, lo cual es concordante con el ambiente estuarino de los sitios.

A diferencia del resto del conjunto óseo, no se evidenciaron huellas de corte, ni fracturas atribuibles a patrones culturales de consumo, sin embargo, el contexto zooarqueológico en el que se encontraban los especímenes hacía suponer un uso alimenticio, de modo que la verificación de esta idea debía constatarse por otras vías.

Solo se detectó un húmero con su extremo distal quemado, proveniente de EA I (Figura 2). En cambio, el sitio Cuchipuy de Chile central, evidencia restos de la misma especie quemados y calcinados, asociados a enterratorios humanos (Bastías 20l I). 


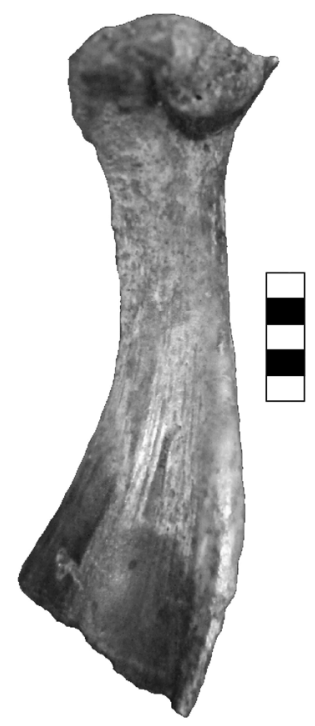

Figura 2: Húmero de rana con extremo distal quemado, escala $5 \mathrm{~mm}$.

Figure 2: Frog humerus, distal end burned, scale $5 \mathrm{~mm}$.

Del mismo modo, se observaron sólo dos marcas atribuibles a masticación humana, probablemente de un premolar y de un incisivo, ambas de RT-39 (Figura 3). Su asignación a marcas de consumo implica descartar otro tipo de huellas, como pisoteo o carroñeo de carnívoros.

El pisoteo (sensu Blasco et al. 2008), más bien produce superficies estriadas aisladas $\circ$ bien formando grupos en patrones o variables en longitud y ancho, cuyos extremos aparecen cortados abruptamente y no muestran ningún estrechamiento que indique direccionalidad. La posición de estas marcas no está relacionada con la estructura anatómica del hueso y su es aleatoria (Blasco et al. 2008:1606).

Las modificaciones presentadas incluyen morfologías difícilmente creadas por carnívoros. El resultado de la acción de carnívoros en estos huesos generalmente deja los bordes cranulados (Blasco et al. 2008:1607). De igual modo, se descartan como huellas dejadas por roedores (Andrew 1990 y 1995 , Haglund et al. 1988).

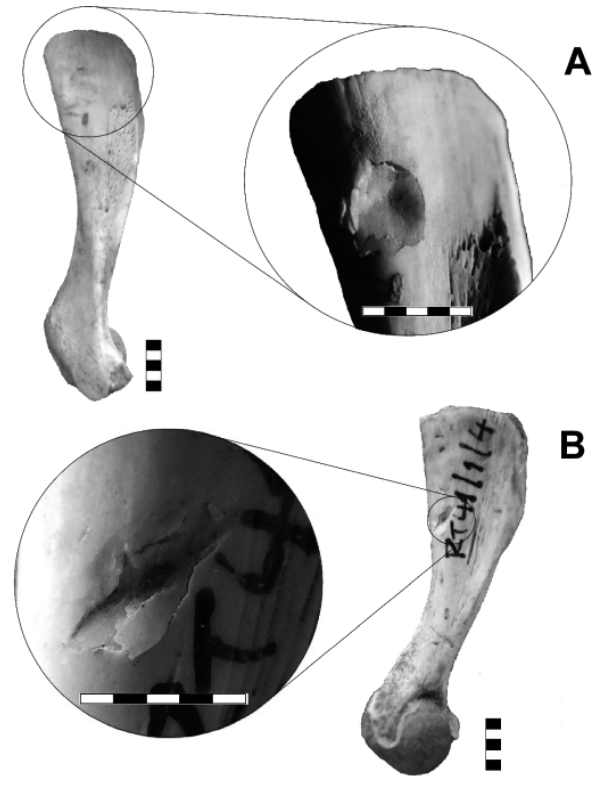

Figura 3: a) Húmero de rana en RT-39, huella atribuible a premolar humano, b) Húmero de rana en RT39, huella atribuible a incisivo humano. Escala $5 \mathrm{~mm}$ (RT-39, sitio arqueológico Raqui Tubul 39).

Figure 3: a) Frog humerus in RT-39, trace attributable to human premolar, b) Frog humerus in RT-39, trace attributable to human incisor. Scale $5 \mathrm{~mm}$.

Como señaló Binford (1981:|48; también Elkin y Mondini 20ll), los carnívoros no tienen monopolio en el mordisqueo, siendo normal que el patrón de consumo de humanos lo incluya. Sin embargo, el mordisqueo humano ha sido escasamente atendido en investigaciones de cazadores recolectores, contrastable a las contribuciones relativas a los carnívoros (Haglund et al. 1988, Cruz-Uribe, I99I, Binford I98I, entre otros), lo que concuerda con el par de huellas observadas en huesos de RT39 (ver Figura 3a y 3b).

Recientes investigaciones sobre mordisqueo humano (Fernández-Jalvo et al. 1999, Landt 2007, Saladié et al.20 I3), han permitido determinar patrones como marcas de corte oblicuas y lascados corticales adheridos en el área de fractura (Fernández-Jalvo et al. 1999), observados en fracturas de huesos frescos (Blasco et al. 2008:16/3). 


\section{CONCLUSIONES}

Tanto la predominancia de patas traseras como las marcas de combustión y mordisqueo humano en los huesos indica que los anfibios eran parte de la dieta de poblaciones asentadas en sitios cercanos a cuerpos de agua. Las huellas no se superponen con otras marcas en los huesos, lo que permitiría una caracterización preliminar de un posible patrón de consumo.

Se deben desarrollar estudios en relación a estrategias procesamiento de anfibios considerando una investigación histórica, experimentación actualística (Borrero 1991) y preparación de ejemplares para conformar colecciones de referencia, las que pueden brindar un espectro más amplio de especies (Sampson 2003), así como la posibilidad de que los sitios estén siendo ocupados por temporadas (Klein 1979). De igual modo se deben refinar los medios de recuperación de la evidencia ósea tanto en el harneo como utilizando flotación (Kiselý 2008).

Finalmente, consideramos que las modificaciones observadas deben ser categorizadas a través de microscopía electrónica de barrido, con el fin de afinar herramientas analíticas que permitan interpretar los ensamblajes, en relación a determinar la naturaleza de los agentes implicados en su acumulación (Pinto y Andrews 1999, Landt 2007).

\section{AGRADECIMIENTOS:}

Este trabajo fue escrito en el marco del Proyecto Fondecyt 1020272 Estrategias adaptativas entre los grupos El Vergel en las costas de la Araucanía.

\section{BIBLIOGRAFÍA}

Andrews, P. 1990. Owls, Caves, and Fossils. The University of Chicago Press, Chicago.

Andrews, P. 1995. "Experiments in Taphonomy". Journal of Archaeological Science 22: 147-I53.

Bastías, J. 20II. Una mirada a la fauna menor del sitio Cuchipuy. Informe de práctica profesional, Departamento de Antropología, Universidad de Chile, manuscrito en posesión del autor.

Binford, L. 198I. Bones: Ancient Men and Modern Myths Academic Press, New York.

Borrero, L.A. 1991. "Experimentos y Escalas Arqueológicas”. Shincal 3(I): |42-| 45.
Blasco, R., J. Rosell, J. Fernández, J. Cáceres, I. \& J.M. Vergès, 2008. "A new element of trampling: an experimental application on the Level XII faunal record of Bolomor Cave (Valencia, Spain)". Journal of Archaeological Science 35: I605-1618.

Cei, J.M. 1962. Batracios de Chile. Ediciones Universidad de Chile, Santiago.

Cruz-Uribe, K. 1991. "Distinguishing Hyena from Hominid Bone Accumulations". Journal of Field Archaeology 18(4): 467-486.

Davis, P. 1997."The Bioerosion of Bird Bones”. International Journal of Osteoarchaeology. 7: 388-40I.

Diaz, H. 2005. Especies de anfibios presentes en Chile. Disponible en http://www2.udec.cl/ hediaz/ (19 de noviembre del 2012).

Diaz, H. \& J. C. Ortiz.2003. "Evaluación del estado de conservación de los anfibios en Chile". Revista Chilena de Historia Natural 76(3): 509-525.

Duellman, W. E. 2003. "Helmeted water toad, Caudiverbera caudiverbera”. En Grzimek's Animal Life Encyclopedia Vol. 6, Amphibians, editado por M. Hutchins, W. E. Duellman \& N. Schlager, p. I 70. Gale Group, Farmington Hills, Michigan.

Duellman, W. E. \& L. Trueb. 1986. Biology of Amphibians. McGraw-Hill Book Co., New York.

Elkin, D. \& M. Mondini. 200I. "Human and Small Carnivore Gnawing Damage on Bones an Exploratory Study and its Archaeological Implications". En Ethnoarchaeology of Andean South America. Contributios to Archaeological Method and Teory, editado por L.A. Kuznar., pp. 255-265. International Monographs in Prehistory. Ethnoarchaeological Series 4,Ann Arbor.

Fernández-Jalvo, Y., J.C. Diez, J.C., I. Cáceres, \& J. Rosell. 1999. "Human cannibalism in the Early Pleistocene of Europe (Gran Dolina, Sierra deAtapuerca, Burgos, Spain)”. Journal of Human Evolution 37: 591-622.

Formas J.R. 1995. Anfibios. En Diversidad biológica de Chile , editado por J.A. Simonetti, M.T.K. Arroyo, A.E. Spotorno \& E. Lozada, pp: 3 | 4-325. Conicyt, Santiago.

Frost, D.H. 2004. Amphibian Species of the World 3.0, an Online Reference. New York, American Museum of Natural History. Disponible en http://research.amnh.org/herpetology/ amphibia (19 de noviembre del 20I2).

Gans, C. \& T. S. Parsons. 1966. "On the origin of the jumping mechanism in frogs”. Evolution 20(I): 92-99.

Haglund, W.D., D.T. Reay, \& D.R. Swindler. 1988. Tooth Mark Artifacts and Survival of Bones in Animal Scavenged Human Skeletons. Journal of Forensic Sciences, JFSCA 33(4): 985-997.

Kyselý, R. 2008. "Frogs as a part of the Eneolithic diet. Archaeozoological records from the Czech Republic (Kutná Hora-Denemark site, Rivnáč Culture)". Journal of Archaeological Science 35: 143-157.

Klein, R.G. 1979. "Paleoenvironmental and Cultural Implications of Late Holocene Archaeological Faunas from the Orange Free State and North-Central Cape Province, South Africa”. The South African Archaeological Bulletin. 34(I29): 34-49.

Landt, M.J. 2007. "Tooth marks and human consumption: ethnoarchaeological mastication research among foragers of the Central African Republic". Journal of Archaeological Science 34: 1629-1640.

Myers, C.W. \& R. B. Stothers. 2006. "The myth of Hylas revisited: the frog name Hyla and commentary on Specimen medicum (1768) of J.N. Laurenti, the "father of herpetology". Archives of natural history 33(2): 24I-266. 
O'Connor, T. 2000. The archaeology of animal bones. Sutton Publishing, Great Britain.

Pinto, A.M. \& P. Andrews. 1999. "Amphibian taphonomy and its application to the fossil record of Dolina (middle Pleistocene, Atapuerca, Spain)". Palaeo 149: 4 I I-429.

Quiroz, D. 2003. Ocupaciones El Vergel en las costas de la Araucanía. Santiago. Actas del $4^{\circ}$ Congreso Chileno de Antropología, tomo II, pp. I456-I465. Santiago, Chile.

Reitz, E.J. \& E.S. Wing. 1999. Zooarchaeology. Cambridge University Press, New York.

Ročková, H. \& Z. Roček. 2005. "Development of the pelvis and posterior part of the vertebral column in the Anura". Journal of Anatomy 206: 17-35.

Roček, Z. 2003. "Larval development and evolutionary origin of the anuran skull”. En Amphibian Biology: Osteology, editado por H. Heatwole, pp: I878-1995. Surrey Beatty \& Sons, Chipping Norton, Australia.

Saladié, P., A. Rodríguez-Hidalgo, C. Diez, Martín-Rodriguez, P. \& E. Carbonell, E. 2013.
"Range of bone modifications by human chewing". Journal of Archaeological Science 40: 380-397.

Sampson, C.G. 2003. "Amphibians from the Acheulan site at Duinefontein 2 (Western Cape, South Africa)". Journal of Archaeological Science 30: 547-557.

Stahl, P.W. 2000. "Archaeofaunal Accumulation, Fragmented Forest, and Anthropogenic Landscape Mosaics in the Tropical Lowlands of Prehispanic Ecuador". Latin American Antiquity I I (3): 24I-257.

Velásquez, H. \& L. Adán. 2002. “Evidencias arqueofaunísticas del sitio alero Marifilo I.Adaptación a los bosques templados de los sistemas lacustres cordilleranos del centro sur de Chile”. Boletín de la Sociedad Chilena de Arqueología 33-34: 27-35.

Yerkes, R.W. 2005. "Archaeology Bone Chemistry, Body Parts, and Growth Marks: Evaluating Ohio Hopewell and Cahokia Mississippian Seasonality, Subsistence, Ritual, and Feasting". American Antiquity 70(2): 24I-265. 